\title{
A new approach to measure the elasticity modulus for ceramics using the deformation energy method ${ }^{\dagger}$
}

\author{
Cesar R. Foschini ${ }^{1}$, Edson A. C. Souza ${ }^{1}$, Ana F. S. Borges ${ }^{2}$ and Carlos A. F. Pintão ${ }^{3, *}$ \\ ${ }^{1}$ Department of Engineering, Feb-UNESP, 17033-360, Bauru, SP, Brazil \\ ${ }^{2}$ Department of Material, FOB-USP, 17012-901, Bauru, SP, Brazil \\ ${ }^{3}$ Department of Physics, FC-UNESP, 17033-360, Bauru, SP, Brazil
}

(Manuscript Received July 20, 2015; Revised January 25, 2016; Accepted April 11, 2016)

\begin{abstract}
This paper presents an alternative method to measure the modulus of elasticity to traction, $E$, for relatively limited sample sizes. We constructed a measurement system with a Force sensor $(F S)$ and a Rotation movement sensor $(R M S)$ to obtain a relationship between force $(F)$ and bending $(\Delta L)$. It was possible by calculating the strain energy and the work of a constant force to establish a relationship between these quantities; the constant of proportionality in this relationship depends on $E, I$ and $L . I$ and $L$ are the moment of inertia of the uniform cross-section in relation to an oriented axis and length, respectively, of the sample for bending. An expression that could achieve the value of $E$ was deduced to study samples of Y-TZP ceramics. The advantages of this system compared to traditional systems are its low cost and practicality in determining $E$.
\end{abstract}

Keywords: Deformation energy; Elasticity modulus; Force sensor; Rotational movement sensor; Y-TZP

\section{Introduction}

Scientific research and development of new materials for implantology is constantly aiming at the perfection of mechanical performance and surface properties. Over $80 \%$ of all implant devices are metals [1,2], although other types of materials are used such as polymers and ceramics. However, there are difficulties in the techniques used for characterization of new materials and the study of its mechanical properties. This work was conducted to measure the modulus of elasticity to traction, $E$, in order to implement an alternative technique for ceramics samples, which have relatively small dimensions and an unknown Poisson coefficient. The methods and techniques for obtaining $E$ usually include four types: Static (tensile, torsion, bending test); dynamic (resonant frequency method); wave propagation (ultrasonic pulse-echo method); and nanoindentation. Each method has its own advantages and disadvantages. Among these, the most commonly used is pulse-echo ultrasound $[3,4]$. This technique is nondestructive and does not alter the sample's physical and chemical properties, making it of great practical interest. However, it can only measure $E$ values for a single sample temperature and well-defined sizes. In such a case, informa-

${ }^{*}$ Corresponding author. Tel.: +55 1431036084, Fax.: +55 1431036095

E-mail address: fonzar@fc.unesp.br

${ }^{\dagger}$ Recommended by Associate Editor Jun-Sik Kim

(C) KSME \& Springer 2016 tion on the Poisson coefficient is needed to obtain $E$, and sample dimensions may be critical. This does not occur with the torsion pendulum technique $[5,6]$, which also permits obtaining $E$ but has an advantage in relation to previous techniques because it is a dynamic measure, without interruption, using a temperature band between 77 and $700 \mathrm{~K}$ [4-7]. However, it must be performed in a vacuum of around $10^{-6} \mathrm{mBar}$ [8] due to the high temperatures into which the sample is submitted. In this case, Poisson coefficient value information is needed to obtain $E$, and sample dimensions may be critical. The TZP ceramics, which have their minimum requirements as implants for surgery described in ISO 13356, are currently the materials of choice by nearly all manufacturers producing femoral head prostheses. More than 300000 TZP hip prostheses have been installed [9]. Zirconium's remarkable behavior is mainly due to the toughening mechanism found in these materials, which is called a martensitic transformation. Zirconium is a polymorphic ceramic with three, well-defined, crystalline allotropic shapes - monoclinic, tetragonal and cubicthat are stable at different temperatures. In its natural state and at ambient temperature, zirconium exhibits monoclinic symmetry and is stable at a temperature of up to approximately $1170^{\circ} \mathrm{C}$. Between $1170^{\circ} \mathrm{C}$ and $2370^{\circ} \mathrm{C}$, zirconium modifies to the tetragonal phase, which has a reduced volume when compared to the monoclinic phase. This phase can be stabilized at ambient temperature depending on the size of the zirconium 
Table 1. Group name, material commercial name, manufacturer and composition of a Y-TZP block.

\begin{tabular}{|c|c|c|c|}
\hline Group & Material & Manufacturer & Composition \\
\hline ZYZ & $\begin{array}{c}\text { In ceramics } \\
\mathrm{ZYZ}\end{array}$ & $\begin{array}{c}\text { Vita Zahnfabrik } \\
\text { (Bad Säckingen, } \\
\text { Switzerland) }\end{array}$ & $\begin{array}{l}-\mathrm{ZrO}_{2}=91.00 \%, \\
\text { Zirconium dioxide } \\
\cdot-\mathrm{Y}_{2} \mathrm{O}_{3}=5.00 \%, \\
\text { Yttrium oxide } \\
\cdot-\mathrm{HfO}_{2} \leq 3.00 \%, \\
\quad \mathrm{Hafnium} \text { oxide } \\
\text { - } \mathrm{Al}_{2} \mathrm{O}_{3}+\mathrm{SiO}_{2} \leq 1.00 \%, \\
\text { Aluminum oxide }+ \text { Silicium } \\
\text { oxide }\end{array}$ \\
\hline
\end{tabular}

particle and the concentration of oxide stabilizers added to its structure. Above $2370^{\circ} \mathrm{C}$ until fusion $\left(2680 \pm 15^{\circ} \mathrm{C}\right)$, zirconium is in the cubic phase [10]. At high temperatures, or after quick cooling to temperatures near or lower than liquid nitrogen, a structure with orthorhombic symmetry can be observed. As we wanted a practical and economic solution for the type of sample used in this work, we decided to invest in the construction of the system described below to determine the elasticity modulus $E$.

\section{Materials and methods}

\subsection{Materials}

The Y-TZP samples used in the elasticity module measurements are described in Table 1 . We initially describe the elastic deflection energy method and then explain how to determine the biomaterials' elasticity module with the system used in this study.

\subsection{Deflection energy method for obtaining $E$}

Consider an elastic structure submitted to applied loads and deflected elastically. In this deflection process, the Principle of Energy Conservation [11] is used, expressed as

$$
W_{E}+Q=\Delta E
$$

$\mathrm{W}_{\mathrm{E}}$ is the work carried out by applied external forces, $Q$ is the heat exchanged by the structure with its surrounding area, and $\Delta E$ is the variation of associated energies - kinetic energy $(K)$ and internal energy $(U)$ - of the structure. Considering that these loads are applied in a gradual and increasing manner and that a state of equilibrium is maintained in this process, then the variation of kinetic energy is zero and $\Delta E$ is only due to the variation in internal energy $U$. Eq. (1) in these conditions is reduced to

$$
W_{E}=\Delta U
$$

The work can be considered as energy stored in the structure due to strain and stress in an infinitesimal volume element [11-17] and which will be represented by strain and stress tensors, $\sigma_{\mathrm{ij}}$ and $\mathrm{e}_{\mathrm{ij}}$, respectively. The energy $d U$ stored in this element when stress has reached its final value $\mathrm{e}_{\mathrm{ij}}$ is

$$
d U=\int_{0}^{e_{i j}} \sigma_{i j} d e_{i j}
$$

By integrating the entire volume $V$ of the structure, we get the total internal energy $U$ due to the deflection expressed as

$$
U=\int\left(\int_{0}^{e_{i j}} \sigma_{i j} d e_{i j}\right) d V
$$

In cases where the elastic structure has a linear behavior and is isotropic and submitted to pure bending using Hooke's Law [11], then

$$
U=\int \frac{1}{2 E} \sigma_{11}^{2} d V+\int \frac{1}{2 G} \sigma_{12}^{2} d V
$$

with $E$ and $G$ being modules of traction and torsion elasticity, respectively.

For measuring $E$, a prismatic bar is considered with a uniform cross section of area $A$ and length $L$, submitted to applied force $F$ using a force sensor at one of the ends and with the other crimped onto, one pure bending, see Figs. 1(a)-(c).

With regard to material resistance [12], it is known that the state of tension at one end of bar $\mathrm{x}_{\mathrm{i}}$, given the experimental conditions of Fig. 1, is expressed as:

$$
\sigma_{11}=-\frac{M x_{2}}{I} \text { and } \sigma_{12}=\frac{F M_{S}}{I b} \text {. }
$$

$I$ represents the moment of inertia [18, 19] of a cross section $A$ in relation to axis $\mathrm{x}_{3}\left(I=\int x_{2}^{2} d a\right)$ and $M_{S}$, 1st order moment in relation to axis $\mathrm{x}_{3}, M_{S}=\int x_{2} d a$. The values of $\sigma_{\mathrm{ij}}$ are zero for other tensions. $M$ and $F$, respectively, represent the bending moment and the shearing force and they act on an arbitrary cross section at $\mathrm{x}_{1} . M$ is a function of $\mathrm{x}_{1}\left(M=F \mathrm{x}_{1}\right)$ and $F$, in this case is a constant for each of the states of static equilibrium. In this case, $M_{S}=\int x_{2} d a=0$, because the centroid of the cross section area coincides with point $\mathrm{O}$. Thus, the term related to $\sigma_{12}$, of Eq. (6) is zero. Replacing these components of tension in Eq. (5), you get the internal energy of deflection in the structure for this application.

$$
U=\frac{F^{2} L^{3}}{6 E I}
$$

The external work conducted by $F$ is

$W_{E}=F \Delta L$.

Equating the Eqs. (7) and (8), you get:

$$
F=\frac{6 E I}{L^{3}} \Delta L=B^{*} \Delta L .
$$


Therefore, slope $B^{*}=6 E I / L^{3}$ is a constant in the elastic region and depends on the nature of the material, the geometry of the uniform cross-section, and the length of the sample, represented by $E, I$ and $L$, respectively.

For two samples of distinct natures 1 and 2, considering the same cross-sectional area $\left(A_{1}=A_{2}\right)$ and length $\left(L_{1}=L_{2}\right)$ with different geometries, their deformed volume will be equal. This is a condition order to be determined $(E)$ for the unknown sample $\left(E_{2}\right)$ in relation to that known and used as reference $\left(E_{1}\right)$. The other condition is that the calibration factor due to the force sensor $f$ has to be the same for each measurement. As the calibration factor depends on the $L$ value, then it must be the same measured length $\left(L_{1}=L_{2}=L\right)$, and consequently the areas of the cross-section but not necessarily the geometries must be the same. In this case, the values of $I$ are generally different $\left(I_{1} \neq I_{2}\right)$ and depend on the geometry of the uniform cross-section. The above describes the situation presented in this study.

If we replace the moment of inertia values for the crosssectional areas $I_{2}=b a^{3} / 12$ (rectangular section) or $I_{1}=$ $\pi D^{4} / 64$ (circular section) and use $B^{*}$ and $f$, we get the following equations for calculating $E$ for the two situations:

$$
\begin{aligned}
& E_{2}=\frac{B_{2}^{*} L^{3} 2 f}{b a^{3}} \text { or } \\
& E_{1}=\frac{B_{1}^{*} L^{3} 32 f}{D^{4} 3 \pi} .
\end{aligned}
$$

In this case, the value $E_{2}$ can be expressed by $E_{2}=$ $E_{1} I_{1} B_{2}^{*} / B_{1}^{*} I_{2}$.

If the geometries (circular or rectangular) and the dimensions are the same for both samples, then the value $I$ will be the same, too. To achieve $E$ would result in the expression $E_{2}=E_{1} B_{2}^{*} / B_{1}^{*}$. The subscripts correspond to samples 1 or 2 .

\subsection{Measurement of the modulus of elasticity $E$}

To measure the turning angle $\varphi(\mathrm{rad})$ of the screw that moves table XY when it shifts from $\Delta L$, a Rotation movement sensor $(R M S)$ was used [20]. A pulley of diameter $\phi=28.70 \pm$ $0.05 \mathrm{~mm}$ was attached to the $R M S$ shaft, and with the belt that passes through the pulley and to another pulley of the same diameter affixed to the shaft of the table transmission mechanism, $\varphi$ can be registered. At the same extremity with the rotation sensor, a microwave motor was adapted with a constant torque in order to move table $\mathrm{XY}$ in the two required directions, one for approximation and the other for drawing it away from the force sensor. At the opposite end of the rotation sensor there is one piece of steel which is attached a sample. This sample has the other free end (see Fig. 1). At a distance $L$ from this free extremity, a force sensor was positioned (FS) affixed to a base on a bench. Upon turning the movement transmission screw for table XY at an angle $\varphi(\mathrm{rad})$, the sample was shifted $\Delta L$, evaluated by $\varphi(0.000160) \mathrm{m}$.

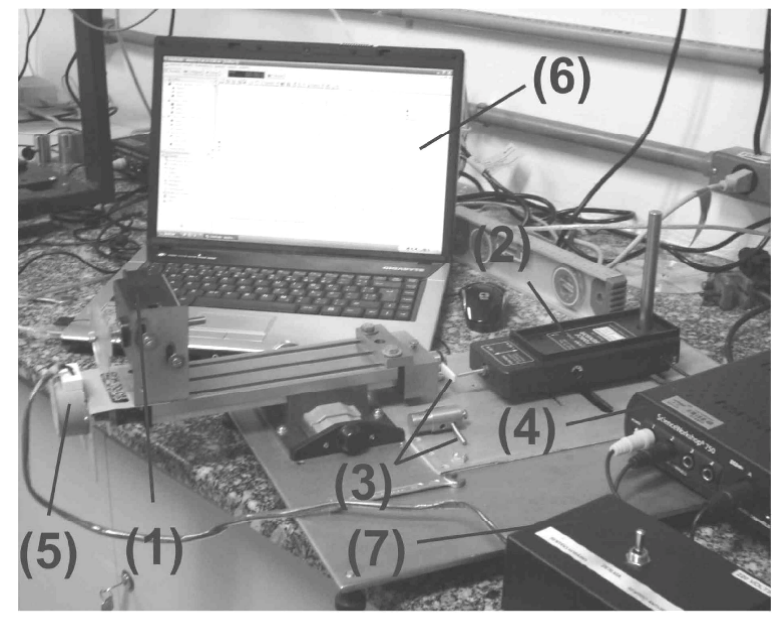

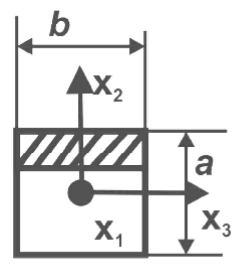

Cross section

(b) (a)

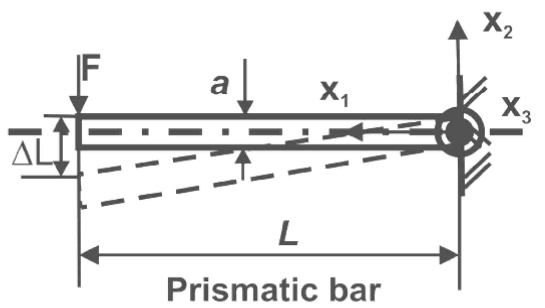

(c)
Fig. 1. (a) System for measuring $E$ : (1) Rotational movement sensor (RMS-PASCO: CI6538; [20]); (2) force sensor (FS-PASCO: CI6537); (3) samples: Titanium and ceramics; (4) interface (PASCO: CI7650750); (5) motor for moving table XY; (6) computer with specific software; (7) key to activate motor; (b) cross-section of sample of area $A$; (c) Prismatic bar of length $L$.

Thus, pure bending was slowly applied to the sample, using force $F$ at the free extremity. $F S$ is zeroed before beginning the measurement, and the value of $F$ is automatically recorded for each angle $\varphi$ with the RMS. The two sensors are connected at an interface and the interface to a computer; using specific software, the experimental points for the $F(\mathrm{~N})$ versus $\varphi(\mathrm{rad})$ greatnesses graph are obtained simultaneously in real time. It was possible to adjust a straight line and obtain its slope because it behaves linearly in a regime where the material behaves elastically. That slope is called $B_{0}$ and its unit is N/rad, which is transformed to $\mathrm{N} / \mathrm{m}$ using the relation $B^{*}=B_{0}$ $(1 / 0.000160)$ since we determined that $1 \mathrm{rad}$ equates $0.000160 \mathrm{~m}$.

For these measurements, it was necessary to calibrate the force sensor, which has a factor $f$ that can be obtained experimentally and which depends on the fixed length $L$. A sample was chosen of commercially pure titanium (TiCp) with an average elasticity module of $E_{1}=103.4 \mathrm{GPa}$ [21]. This sample had a circular cross-section and was used as a reference for obtaining $E$ from samples of the Y-TZP. The latter samples had a rectangular uniform cross-section. Different $L$ values were selected for the reference sample (TiCp). For each fixed $L$, using three Force $(F)$ curves as a function of the angle $(\varphi)$, the average value for $B_{0}$ was determined. In order to determine 
$f$ of the reference sample, Eq. (11) was used by replacing $B^{*}$ for $B_{0}(1 / 0.000160), D=(3.20 \pm 0.05) \mathrm{mm}$ and $E_{1}=103.4$ GPa. Finally, with Eq. (10), with the experimental results $B_{0}, L$, $a$ and $b$ for the Y-TZP samples, and using the same calibration factor obtained for TiCp, $E\left(E_{2}\right)$ was obtained.

Twelve samples were studied for the ZYZ material group, and then the error associated with $E$ was evaluated, using the statistical theory of errors [22] with a $95 \%$ degree of confidence. From the theory of small samples in statistics, the elasticity modulus $E\left(E_{95 \%}\right)$ was expressed as $E_{95 \%}=E_{M} \pm$ $t_{95 \%} \frac{S}{\sqrt{N-1}}$, where $E_{\mathrm{M}}$ is the average of $N$ values of $E$ from Table 2, and the number $\mathrm{t}_{95 \%}=1.80(N=12)$ is obtained from the student distribution (see attachment III of Ref. [22]), considering a $95 \%$ probability for $E$ to belong to the estimated interval; $S$ is the average squared deviation, which is expressed as $S=\sqrt{\frac{\left(E_{M}-E_{j}\right)^{2}}{N}}$; with $N=12$ and $E_{\mathrm{j}}$ as the $E$ values in the 1 to $N$ interval.

\subsection{Calibration curves and the ffactor}

The $f$ calibration factor, as previously explained, was obtained for the sample that served as a reference (TiCp) and was subsequently used to obtain $E$ in a comparative manner for the Y-TZP samples. A length $L_{1}=L_{2}=5.70 \mathrm{~mm}$ was chosen because the Y-TZP samples had a small length and $f$ to measure the force could not depend on $L$. The samples had a different nature and geometry, and their deformed volumes had to be the same. Therefore, the cross-sectional areas of the Y-TZPs $\left(A_{1}=a b\right)$ should be equal to that of TiCp $\left(A_{2}=\pi D^{2} / 4\right)$ In fact, these areas were approximately equal, with a percentage deviation of $0.5 \%$. To determine the mean values of $B_{0}$ as a function of length $L$ for the TiCp sample, three curves of $F$ as a function of $\varphi$ for each fixed $L$ were calculated. Then, using Eq. (11) and replacing $B^{*}$ for $B_{0}(1 / 0.000160), D=(3.20 \pm$ $0.05) \mathrm{mm}$, and $E_{1}=103.4 \mathrm{GPa}$, the $f$ value was determined for each $L$.

\section{Experimental results}

The results in Fig. 2 show the TiCp calibration curve with the $B_{0}$ mean values and the $f$ factors for each $L$. Of note is that $f$ and $B_{0}$ depend on $L$. Fig. 3 shows the results of a typical curve of $F$ versus $\varphi$ for $L=5.70 \mathrm{~mm}$ for TiCp. Fig. 3 also shows the experimental results for the slope of the typical curves of $F$ versus $\varphi$ for TiCp, which was the reference sample and the length used to determine the value of $E$ for the YTZPs. The calibration factor $f=16.89$ was calculated using Eq. (11) as explained in Subsec. 2.4; it was also obtained from Fig. 2. Fig. 4 shows a typical result from one of $12 \mathrm{Y}$-TZP samples, specifically the $3 \mathrm{~A} Z \mathrm{ZYZ}$, with $L=5.70 \mathrm{~mm}$. Finally, with Eq. (10), with the experimental results $B_{0}, L, a$ and $b$ for the YTZP samples, and using the same calibration factor $f=16.89$ for TiCp, $E\left(E_{2}\right)$ was obtained. In this case, the value $E_{2}$ can be

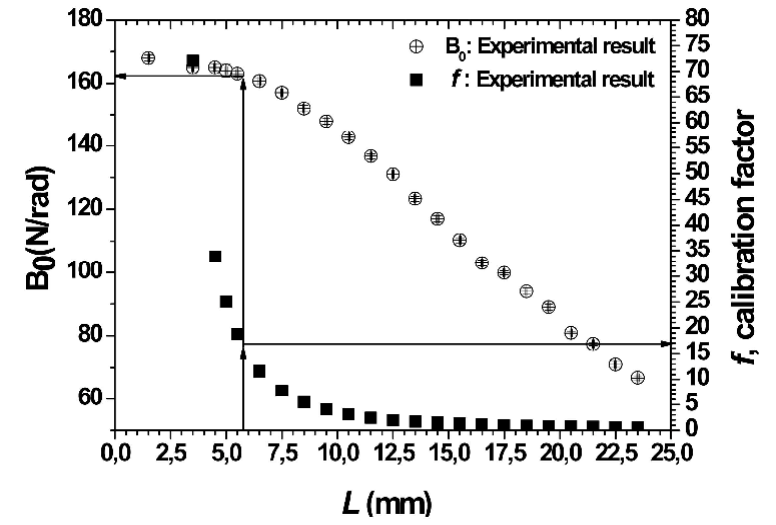

Fig. 2. Calibration curve for the TiCp sample, diameter $D=3.20 \pm$ $0.05 \mathrm{~mm}$, uniform circular section. The calibration factor in Eq. (10), $f$ $=16.89$, was used to obtain $E$ of the Y-TZPs samples with length $L=$ $5.70 \pm 0.05 \mathrm{~mm}$.

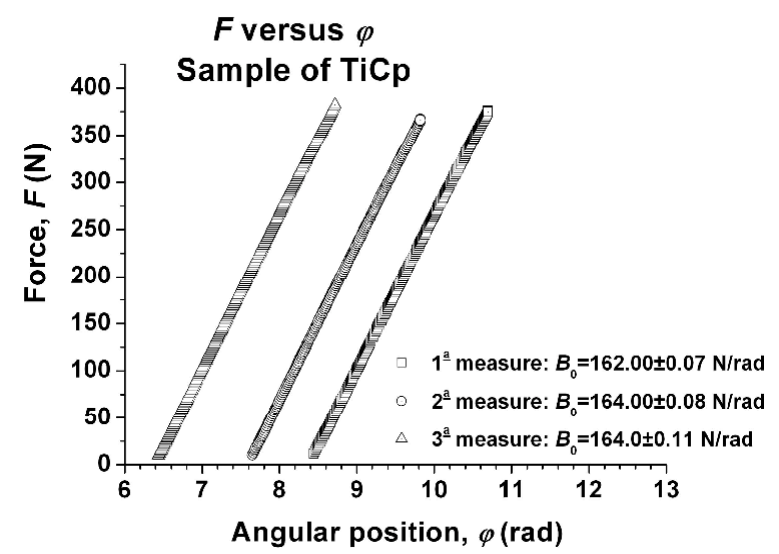

Fig. 3. Typical force curve, $F(\mathrm{~N})$, applied by $F S$ as a function of angular position, $\varphi(\mathrm{rad})$, measured by an $R M S$. Sample of TiCp: $D=3.20$ $\mathrm{mm} ; L=5.70 \mathrm{~mm}$ and $E_{1}=103.4 \mathrm{GPa}$.

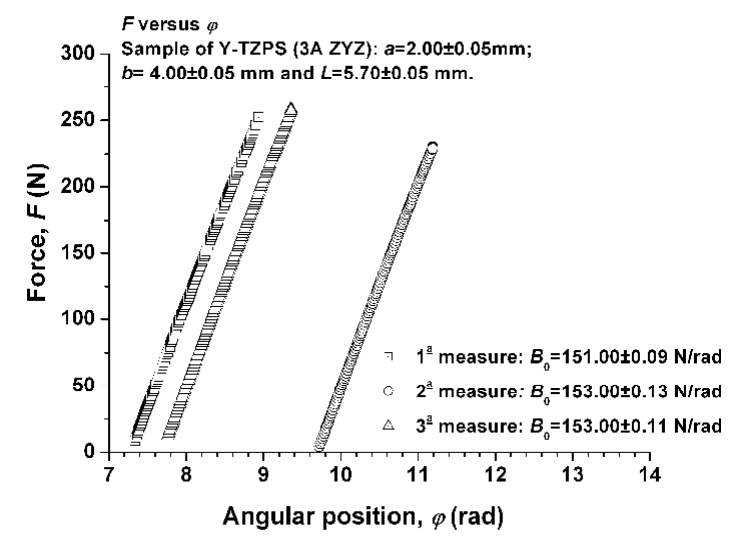

Fig. 4. Typical force curve, $F(\mathrm{~N})$, applied by $F S$ as a function of angular position, $\varphi(\mathrm{rad})$, measured by an $R M S$. Sample of Y-TZP (3A $\mathrm{ZYZ}): a=2.00 \pm 0.05 \mathrm{~mm} ; b=4.00 \pm 0.05 \mathrm{~mm}$ and $L=5.70 \mathrm{~mm}$.

determined directly by $E_{2}=E_{1} I_{1} B_{2}^{*} / B_{1}^{*} I_{2}$. All other Y-TZP results can be seen on Table 2. Fig. 5 shows the $E$ values for each of the studied samples and in relation to the group ZYZ. 
Table 2. Results of the ZYZ samples with reference to TiCp.

\begin{tabular}{|c|c|c|c|c|c|}
\hline Samples & $L(\mathrm{~mm})$ & $a(\mathrm{~mm})$ & $b(\mathrm{~mm})$ & $B_{0}(\mathrm{~N} / \mathrm{rad})$ & $E(\mathrm{GPa})$ \\
\hline $7 \mathrm{~A}$ & 5.70 & 2.00 & 4.00 & $151 \pm 13$ & $184 \pm 22$ \\
\hline $6 \mathrm{~A}$ & 5.70 & 2.20 & 4.00 & $142 \pm 8$ & $130 \pm 12$ \\
\hline $5 \mathrm{~A}$ & 5.70 & 2.00 & 4.15 & $149 \pm 6$ & $176 \pm 16$ \\
\hline $4 \mathrm{~A}$ & 5.70 & 2.20 & 4.00 & $157.7 \pm 0.6$ & $145 \pm 11$ \\
\hline $9 \mathrm{~A}$ & 5.70 & 2.15 & 4.00 & $140.7 \pm 0.6$ & $138 \pm 11$ \\
\hline $3 \mathrm{~A}$ & 5.70 & 2.00 & 4.00 & $152.0 \pm 0.1$ & $186 \pm 15$ \\
\hline $3 \mathrm{~B}$ & 5.70 & 2.10 & 4.00 & $157 \pm 9$ & $166 \pm 17$ \\
\hline $4 \mathrm{~B}$ & 5.70 & 2.00 & 4.15 & $155 \pm 4$ & $183 \pm 15$ \\
\hline $4 \mathrm{C}$ & 5.70 & 2.00 & 4.00 & $151 \pm 2$ & $185 \pm 15$ \\
\hline $6 \mathrm{C}$ & 5.70 & 2.10 & 4.15 & $160 \pm 2$ & $163 \pm 13$ \\
\hline 10B & 5.70 & 2.00 & 4.10 & $154 \pm 4$ & $184 \pm 16$ \\
\hline $10 \mathrm{~A}$ & 5.70 & 2.00 & 4.10 & $152 \pm 2$ & $181 \pm 15$ \\
\hline $\begin{array}{c}\text { TiCp: Circular } \\
\text { section: } D=3.20 \\
\pm 0.05 \mathrm{~mm}\end{array}$ & 5.70 & & & $163.3 \pm 0.09$ & $103 \pm 7$ \\
\hline
\end{tabular}

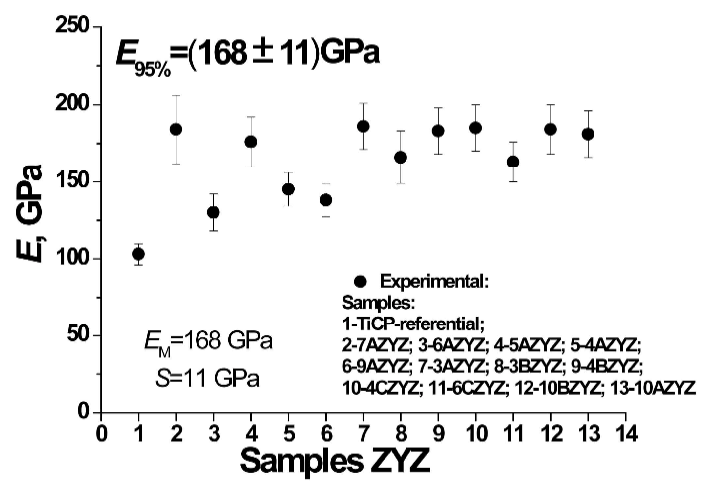

Fig. 5. Values of $E(\mathrm{GPa})$ for the ZYZ samples with reference to TiCp.

Table 2 provides values that are more specific, whereas Fig. 5 presents the $E$ values with the respective experimental errors for the 12 samples studied for the ZYZ group. The errors for each of these samples were evaluated using a first-order approximation of the propagation of uncertainties equation [23]. The value $\mathrm{E}_{95 \%}=168 \pm 11 \mathrm{GPa}$ was obtained using the statistical theory for small samples, as explained in Subsec. 2.3.

\section{Conclusion}

The measurement system presented is an alternative method for obtaining the modulus of elasticity to traction of materials. We applied this system to Y-TZP samples, which have relatively small dimensions and which can be used in other types of materials. Results of the ZYZ group of samples, with a statistical confidence level of $95 \%$, presented an $E$ value equal to $168 \pm 11 \mathrm{GPa}$. The measurement technique employed uses the internal energy of deflection and pure bending. Results corresponded to the expected values as samples similar to pure zirconium have a modulus of elasticity value of $E=203 \pm 14$ $\mathrm{GPa}$, obtained using the ultrasound pulse-echo technique.

\section{Acknowledgements}

This work was supported by FAPESP, proc. 2007/04094-9, from the Department of Physics, UNESP-Campus de Bauru, Brazil.

\section{Nomenclature}

F $\quad$ : Force

$\Delta L \quad$ : Bending

$L \quad$ : Length of prismatic bar

a : Width ceramic sample

b : Thickness of ceramic sample

A : Uniform cross-section

$D \quad$ : Diameter of sample TiCp

$\phi \quad$ : Diameter of pulley

$\varphi \quad$ : Angular position

$V \quad$ : Volume

TiCp : Sample of commercially pure titanium

Y-TZP : Sample of ceramic

$N \quad$ : Number of samples

$\sigma_{i j} \quad:$ Strain tensor

$e_{i j} \quad:$ Stress tensor

$E, E_{\mathrm{j}} \quad$ : Modulus of elasticity to traction

$E_{1}, E_{2}$ : Modulus $E$ of samples 1 and 2 (TiCp and Y-TZP, respectively)

$E_{M} \quad$ : Average of $N$ values of $E_{\mathrm{j}}$

$E_{95 \%} \quad$ : Modulus $E$ of sample Y-TZP, estimated with $95 \%$ probability for $E$ to belong to the estimated interval

$S \quad$ : Average squared deviation

$t_{95 \%} \quad$ : Factor from Student distribution, with $95 \%$ probability for $E$ to belong to the estimated interval

$B_{0} \quad:$ Values related to the average of three slopes in $\mathrm{N} / \mathrm{rad}$

$B^{*} \quad$ : Values related to $B_{0}$ in $\mathrm{N} / \mathrm{m}$

$f \quad$ : Calibration factor

$G \quad$ : Modulus of elasticity to torsion

I : Moment of inertia of cross-section

$I_{1}, I_{2} \quad$ : Moment of inertia of area to sample 1 and 2 (Ti Cp and Y-TZP), respectively

$M_{S} \quad:$ First-order moment in relation to axis

$M \quad$ : Bending moment

$W_{E} \quad$ : Work carried out by applied external forces

$Q \quad$ : Heat exchanged by the structure

$U \quad$ : Internal energy

$K \quad$ : Kinetic energy

\section{References}

[1] T. Hanawa, Research and development of metals for devices based on clinical needs, Science and Technology of Advanced Materials, 11 (6) (2012) 64102-64116.

[2] M. Niinomi, M. Nakai and J. Hieda, Development of new metallic alloys for biomedical applications, Acta Biomaterialia, 8 (11) (2012) 3888-3903.

[3] R. Truel, C. Elbaum and B. B. Chick, Ultrasonic methods in Solid State Physics, Academic Press: New York (1969) 136- 
138.

[4] A. S. Nowick and B. S. Berry, Anelastic relaxation in crystalline solids, Academic Press, New York (1972).

[5] C. A. F. Pintão, L. H. Almeida and C. R. Grandini, Medida do Momento de inércia de um pêndulo de torção para estudo de relaxações anelásticas, Revista Brasileira de Aplicações de Vácuo, 25 (4) 189-192 (2006).

[6] C. R. Grandini, L. H. Almeida and C. A. F. Pintão, Módulo de elasticidade dinâmico de ligas de Ti usadas como biomaterial, $17^{\circ}$ CBECIMat (2006) 4389-4397.

[7] ISO 6721-2, Plastics - Determination of dynamic mechanical properties - Part 2: Torsion - pendulum method, Switzerland (1994).

[8] C. R. Grandini, A low cost automatic system for anelastic relaxations measurements, Revista Brasileira de Aplicações de Vácuo, Campinas, Brasil, 21 (1-2) (2002) 13-16.

[9] J. Chevalier, J. M. Drouin and B. Cales, Low temperature ageing behaviour of zirconia hip joint heads, L. Sedel and C. Rey (editors), Bioceramics, Amsterdam, Elsevier (1977) 135-137.

[10] P. Vena, D. Gastaldi, R. Contro and L. Petrini, Finite element analysis of the fatigue crack growth rate in transformation toughening ceramics, International Journal Plastic, 22 (5) (2006) 895-920.

[11] T. R. Tauchert, Energy principles in structural mechanics, Nova York, McGraw-Hill (1974).

[12] S. P. Timoshenko and J. N. Goodier, Teoria da elasticidade, $3^{\text {a }}$ Ed., Rio de Janeiro: Guanabara Dois (1980).

[13] I. H. Shames and F. A. Cozzareli, Elastic and inelastic Stress analysis, Prentice-Hall International, Englewood Cliffs, New Jersey (1992).

[14] A. P. Boresi and K. P. Chong, Elasticity in engineering mechanics, $2^{\text {a }}$ Ed., John Wiley \& Sons (1999).

[15] T. M. Charton, Energy Principles in theory of structures, Oxford University Press (1973).
[16] H. L. Langhar, Energy methods in applied mechanics, $2^{\text {nd }}$ Edition, Krieger Pub. Co. (1989).

[17] I. N. Reddy, Energy principles and variational methods in applied mechanics, John Wiley \& Sons (2002).

[18] F. P. Beer and E. R. Johnston Jr., Vector mechanics for engineers, Statics, $5^{\text {th }}$ Edition, McGraw-Hill, New York (1992).

[19] J. L. Synge and B. Griffith, Principles of mechanics, $2^{\text {nd }}$ Ed., McGraw-Hill, New York (1949).

[20] PASCO Scientific User's Guide, Science Workshop Version 2.2, Roseville, CA, USA (1996) 285.

[21] M. Long and H. J. Rack, Titanium alloys in total joint replacement-a materials science perspective, Biomaterials, 19 (1998) 1621-1639.

[22] M. R. Spiegel, Estatística, 4th Ed., Rio de Janeiro: Editora McGraw-Hill do Brazil, Ltd. (1972) 312.

[23] J. H. Vuolo, Fundamentals of the theory of errors, 2nd Ed., São Paulo: Editora Edgard Blücher Ltda (1996).

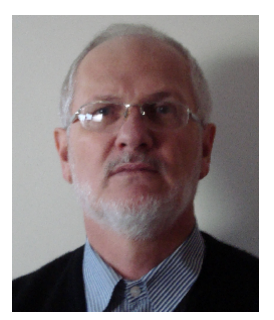

Carlos Alberto Fonzar Pintão was born in Bilac, Brazil in 1954. He received B.Sc. degree in Physics and Mechanical Engineering from the São Paulo University (USP) and the Faculty of Industrial Engineering (FEI), Brazil in 1985 and 1979, respectively, the M.Sc. degree from the São Paulo University (USP), São Carlos, Brazil in 1989 and the Ph.D. degree from the São Paulo University (USP), São Carlos, Brazil in 1996. During the last 28 years he has lectured Physics for Engineering and Physics courses. Most of his research is focused on electron secondary emission in metals and polymers, mechanical properties of materials (elastic modulus and internal friction) and physics experiments in education. 